\title{
Simultaneous Cationic and Anionic Ligand Exchange For Colloidally Stable CsPbBr3 Nanocrystals
}

Muhammad Imran, Palvasha. ljaz, Luca Goldoni, Daniela Maggioni, Urko

Petralanda, Mirko Prato, Guilherme Almeida, Ivan Infante, and Liberato Manna

ACS Energy Lett., Just Accepted Manuscript • DOI: 10.1021/acsenergylett.9b00140 • Publication Date (Web): 04 Mar 2019

Downloaded from http://pubs.acs.org on March 5, 2019

\section{Just Accepted}

"Just Accepted" manuscripts have been peer-reviewed and accepted for publication. They are posted online prior to technical editing, formatting for publication and author proofing. The American Chemical Society provides "Just Accepted" as a service to the research community to expedite the dissemination of scientific material as soon as possible after acceptance. "Just Accepted" manuscripts appear in full in PDF format accompanied by an HTML abstract. "Just Accepted" manuscripts have been fully peer reviewed, but should not be considered the official version of record. They are citable by the Digital Object Identifier (DOI®). "Just Accepted" is an optional service offered to authors. Therefore, the "Just Accepted" Web site may not include all articles that will be published in the journal. After a manuscript is technically edited and formatted, it will be removed from the "Just Accepted" Web site and published as an ASAP article. Note that technical editing may introduce minor changes to the manuscript text and/or graphics which could affect content, and all legal disclaimers and ethical guidelines that apply to the journal pertain. ACS cannot be held responsible for errors or consequences arising from the use of information contained in these "Just Accepted" manuscripts. 


\title{
Simultaneous Cationic and Anionic Ligand Exchange For Colloidally Stable $\mathrm{CsPbBr}_{3}$ Nanocrystals
}

\author{
Muhammad Imran $^{\dagger}$, Palvasha Ijaz ${ }^{\dagger}{ }^{\Delta}$, Luca Goldoni ${ }^{\perp}$, Daniela Maggioni $^{\ddagger}$, Urko Petralanda ${ }^{\dagger}$, Mirko \\ Prato $^{\Phi}$, Guilherme Almeida $^{\dagger}$, Ivan Infante ${ }^{\S * *}$ and Liberato Manna ${ }^{\dagger *}$ \\ ${ }^{\dagger}$ Department of Nanochemistry, ${ }^{\perp}$ Analytical Chemistry Facility and ${ }^{\Phi}$ Materials Characterization Facility, Istituto Italiano \\ di Tecnologia, Via Morego 30, 16163 Genova, Italy \\ ${ }^{\Delta}$ Dipartimento di Chimica e Chimica Industriale, Università degli Studi di Genova, Via Dodecaneso 31, 16146 Genova, \\ Italy \\ ‡ Dipartimento di Chimica, Università degli Studi di Milano, Via Golgi 19, 20133 Milano, Italy \\ $\S$ Department of Theoretical Chemistry, Faculty of Science, Vrije Universiteit Amsterdam, de Boelelaan 1083, 1081 HV \\ Amsterdam, The Netherlands
}

\begin{abstract}
Post-synthesis ligand exchange has been employed extensively on lead halide perovskite (LHP) nanocrystals (NCs), but the complex ligand shell composition of the starting NCs prevented a clear understanding of the exchange process and the surface chemistry of the final NCs remained poorly characterized. Here, we describe a ligand exchange strategy involving the displacement of both cationic and anionic ligands on native model systems of $\mathrm{CsPbr}_{3} \mathrm{NCs}$, which are exclusively coated with Cs-oleate. These ligands are exchanged with various quaternary ammonium bromides $\left(\mathrm{R}_{4} \mathrm{NBr}\right)$ and complete exchange is confirmed by nuclear magnetic resonance (NMR) spectroscopy analysis. The displacement of the native Cs-oleate ligands with proton-free $\mathrm{R}_{4} \mathrm{NBr}$ delivers NCs with excellent colloidal stability and near-unity PLQY, which is preserved after washing with polar solvents and over three weeks of storage in air, and after heating a solution of NCs to $80^{\circ} \mathrm{C}$, as confirmed by NMR analysis. The results, together with density functional theory calculations, suggest that the higher stability of quaternary ammonium capped NC is not due to a stronger binding interaction to the surface, but rather to weaker solventligand interactions of $\mathrm{R}_{4} \mathrm{NBr}$ compared to Cs-oleate, driving the former to the surface of the NC.
\end{abstract}

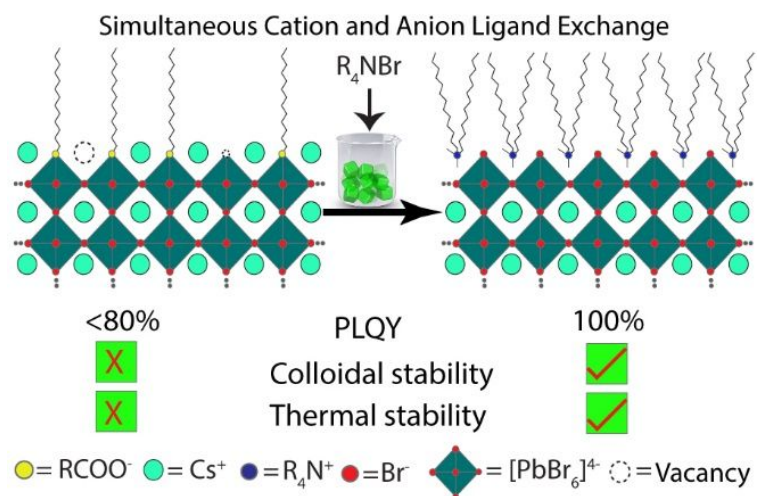

Lead halide perovskites (LHPs) are promising materials for many applications, as they exhibit a tunable band gap in the range of 1.4 to $2.9 \mathrm{eV}$ and an efficient photoluminescence characterized by narrow emission linewidths..$^{1-6}$ Although LHPs are generally considered to be defect tolerant, appropriate surface passivation is still necessary in order to obtain highly efficient and stable emitters. Colloidal LHP nanocrystals (NCs) represent an excellent platform to study the affinity of ligands towards the surface of LHPs and the effects of surface passivation on their light emitting properties. Recent studies have shown that LHP NCs are terminated by a $\mathrm{PbX}_{2}(\mathrm{X}=\mathrm{Cl}, \mathrm{Br}, \mathrm{I})$ layer $^{7}$, passivated by an "AB" layer composed of pairs of charged $\left(\mathrm{A}^{+}\right.$and $\left.\mathrm{B}^{-}\right)$ligands $\mathrm{s}^{8}$, that accommodate on the NC surface by filling the perovskite lattice sites. ${ }^{7}$ These charged ligands may be typical organic molecules that confer colloidal stability to the NC but can also be small ions. For instance, LHP NCs of first generation are passivated by B- ligands such as long chain carboxylates and small halides, in combination/paired with $\mathrm{A}^{+}$ligands such as long chain primary ammoniums and possibly small ions.$^{8-9}$ As far as the electronic properties are concerned, the passivation of surface lead atoms with anionic ligands ( $\mathrm{B}^{-}$) is crucial to 
obtain near-unity photoluminescence quantum yields (PLQYs) while the cationic $\left(\mathrm{A}^{+}\right)$ligands act as spectators.

LHP NCs of first generation typically exhibit PLQYs below unity due to poor surface coverages. Post-synthetic treatments with primary ammonium halides and inorganic salts containing pseudohalide anions $\left(\mathrm{SCN}^{-}, \mathrm{BF}_{4}^{-}\right)$allow to increase the surface coverage and achieve near-unity PLQYs. ${ }^{10-12}$ However, primary ammonium and carboxylate ligands tend to desorb from the NC surface and primary ammonium ions are additionally prone to dissociate into neutral species $\left(\mathrm{RNH}_{3} \mathrm{X} \leftrightarrow \mathrm{RNH}_{2}+\mathrm{HX}_{(\mathrm{g})}\right) \cdot{ }^{13}$ As a consequence, these NCs typically exhibit poor robustness towards washing with polar solvents and their colloidal stability and luminescence properties can only be maintained in the presence of an excess of ligands. This is undesirable in view of further processing of NCs into devices. Therefore, efforts have been made to identify ligands that can provide ideal electronic passivation and bind more tightly to the NCs. For instance, B- ligand exchange of carboxylates with bidentate dicarboxylates or with softer anions such as phosphonates ${ }^{14-15}$ was found to increase the long-term stability of LHP NCs. Nevertheless, the weakly bound primary ammonium are most likely still present in the ligand shell and the robustness of the NCs towards washing steps has not been thoroughly assessed. An alternative was provided by zwitterionic ligands such as sulfobetaine derivatives, which enhance the colloidal stability of $\mathrm{CsPBBr}_{3} \mathrm{NCs}$ and make them withstand washing with polar solvents, but their PLQY remained below $80 \%$, suggesting that this particular surface passivation is still not optimal. ${ }^{16}$
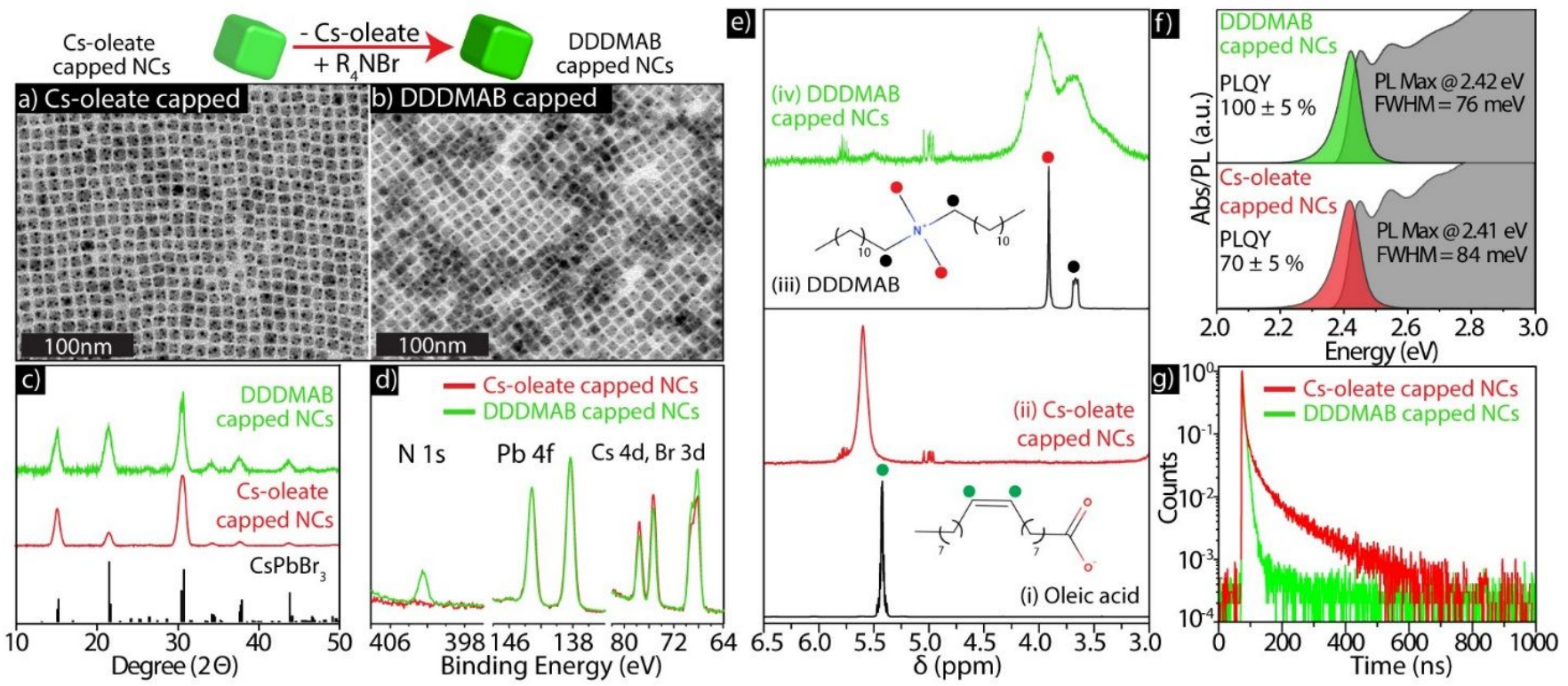

Figure 1. Top left: ligand exchanged scheme using DDDMAB. TEM images of (a) Cs-oleate capped and (b) DDDMAB capped CsPbBr ${ }_{3}$ NCs. (c) XRD patterns of the corresponding samples and reference pattern (COD 96-451-0746). (d) XPS spectra of Cs-oleate capped and DDDMAB capped NCs (normalized to Pb $4 \mathrm{f}$ signals). (e) ${ }^{1} \mathrm{H}$ NMR in toluene- $\mathrm{d}_{8}$ of (i) oleic acid, (ii) Cs-oleate capped $\mathrm{CsPbBr}_{3} \mathrm{NCs}_{\text {, }}$ (iii) DDDMAB (iv) DDDMAB capped CsPbBr 3 NCs. (f) Absorbance and PL spectra of Cs-oleate capped and DDDMAB-capped $\mathrm{CsPbBr}_{3}$ $\mathrm{NC}$ dispersions in toluene and their (g) PL decays.

The "Cs-oleate capped" CsPbBr ${ }_{3} \mathrm{NCs}$ (8.8 nm edge length) used for the ligand exchange reported here were prepared
Here, starting with $\mathrm{CsPBBr}_{3} \mathrm{NCs}$ exclusively capped by Csoleate, ${ }^{17}$ we demonstrate the simultaneous exchange of both $\mathrm{B}^{-}$and $\mathrm{A}^{+}$ligands with various quaternary ammonium bromides which are proton-free and therefore stable, unlike primary ammonium bromides. We show that the treatment of $\mathrm{CsPbBr}_{3} \mathrm{NCs}$ with $\mathrm{R}_{4} \mathrm{NBr}$ molecules where two R groups are long alkyl chains and two others are methyl groups, delivers NCs with excellent colloidal stability and nearunity PLQY, which is preserved after washing with polar solvents and over three weeks of storage in air. According to nuclear magnetic resonance (NMR) spectroscopy, the improved stability and optical properties are due to the strong binding affinity of $\mathrm{R}_{4} \mathrm{NX}$ to the NC surface. Strikingly, DFT calculations indicate that the ligand to NC interaction for $\mathrm{R}_{4} \mathrm{NBr}$ and Cs-oleate are similar in terms of binding energy, even in presence of explicit ligand-ligand interactions, suggesting that the higher colloidal stability of quaternary ammonium bromide coated NCs should be traced back to weaker interactions of the $\mathrm{R}_{4} \mathrm{NBr}$ molecules with the solvent, which is not included in the DFT models.

Although in previous works $\mathrm{R}_{4} \mathrm{NXs}$ have been already reported to improve the performance of LHP thin-film solar cells ${ }^{18}$ as well as the luminescence of first generation NCs up to about $80 \%,{ }^{12,19-20}$ the complex ligand shell composition of the starting NCs prevented a clear experimental investigation of the pre- and post-ligands exchanged $\mathrm{NC}$ surface. The novelty of the present work stands therefore in a clear demonstration of simultaneous anion and cation ligand exchange on LHP NCs, as the surface passivation of the initial and final NCs have been thoroughly assessed. following our recent work, ${ }^{17}$ which employs oleic acid and a secondary amine in the synthesis. As discussed in that work, 
secondary ammonium ions are not part of the NCs ligand shell. ${ }^{17}$ One fraction of this sample was purified and kept as a reference. The remaining crude solution of NCs was mixed with a toluene solution of $\mathrm{R}_{4} \mathrm{NBr}$. For the latter, two $\mathrm{R}$ groups were always methyl, while the other two were either dioctyl $\left(\mathrm{C}_{8}\right)$, didecyl $\left(\mathrm{C}_{10}\right)$ or didodecyl $\left(\mathrm{C}_{12}\right)$. An excess of ethyl acetate was added, and the NCs were separated by centrifugation and redispersed in toluene, see Supporting Information (SI) for further details. We discuss first the case in which $\mathrm{R}_{4} \mathrm{NBr}$ is didodecyl dimethyl ammonium bromide (DDDMAB, $\mathrm{C}_{12}$ ). The oleate capped NCs and those that were ligand exchanged with DDDMAB had the same morphological and structural characteristics, as seen from transmission electron microscopy (TEM, Figure 1a-b) and $\mathrm{X}$-ray diffraction (XRD, Figure $1 \mathrm{c}$ ). The reaction is actually an exchange of Cs-oleate with the ammonium bromide. Elemental analysis via X-ray photoelectron spectroscopy (XPS) revealed that, upon exchange, the $\mathrm{Cs:Pb}$ ratio decreased from 0.9 to 0.8 while the $\mathrm{N}: \mathrm{Pb}$ and the $\mathrm{Br}: \mathrm{Pb}$ ratios increased from 0 to 0.4 and from 2.2 to 2.8 , respectively (Figure $1 \mathrm{~d}$ ). These values suggest that our ligand exchange reaction induced an apparently unbalanced replacement of $0.1 \mathrm{Cs}^{+}$with 0.4 ammonium ions per each $\mathrm{Pb}$ in the NCs. However, it should be noted that the initial Cs-oleate capped NCs do not present a completely passivated outer shell, given that their PLQYs are always below $80 \% .{ }^{17}$ We therefore explain the apparent unbalance with the fact that the quaternary ammonium salts could both exchange native Cs-oleate ligands and also anchor to the vacant sites at the outer shell to completely passivate it. Given the stoichiometry of the used $\mathrm{R}_{4} \mathrm{NBr}$ ligands, the addition of $0.4 \mathrm{~N} / \mathrm{Pb}$ should result in the addition of 0.4 $\mathrm{Br} / \mathrm{Pb}$ as well, while our XPS data indicate an increase of 0.6 $\mathrm{Br} / \mathrm{Pb}$. It is however known that halides have a tendency to desorb from lead halide perovskites when these materials are exposed to ionizing radiations as X-rays or e-beams. ${ }^{21}$ This appears to be even more evident in underpassivated NCs, as should be the case for our starting Cs-oleate capped NCs, where a $\mathrm{Br} / \mathrm{Pb}$ ratio of 2.2 seems to be highly unrealistic (values close to 2.8 have been calculated for NCs of this size $)^{7}$ and is most likely due to high loss of $\mathrm{Br}$ during the XPS experiment itself. The quantification of $\mathrm{Br}$ is therefore underestimated, especially for the Cs-oleate
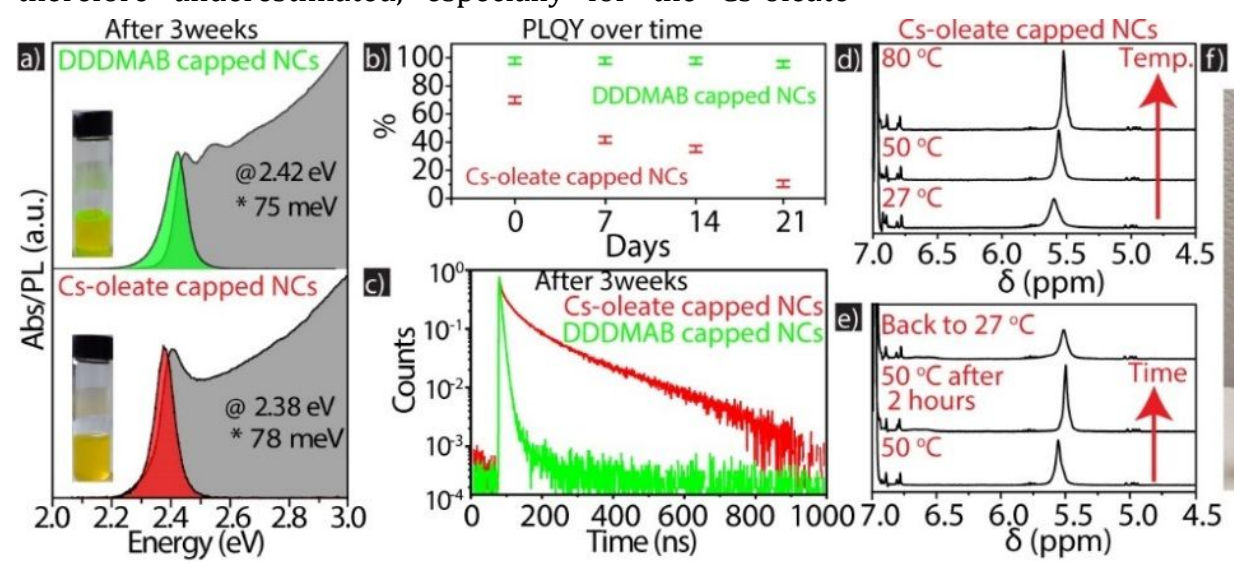

capped NCs, and consequently the obtained 0.6 increase in the $\mathrm{Br} / \mathrm{Pb}$ ratio is likely overestimated.

The ${ }^{1} \mathrm{H}-\mathrm{NMR}$ resonances of the Cs-oleate and DDDMAB capped $\mathrm{CsPbBr}_{3} \mathrm{NCs}$, as well as the respective "free" molecules are reported in Figure 1e. Here, the spectra of the capped NCs exhibit a much broader signal compared to the free molecule cases, due to the binding of ligands to the surface of NCs (complete assignments of resonances are reported in Figures S1-4). The resonances in the DDDMAB capped NCs are ascribable mainly to DDDMAB, with only weak signatures from the oleate molecules. To determine the ligand composition in the DDDMAB treated sample, we dissolved the NCs in deuterated dimethyl sulfoxide and performed quantitative NMR ( $q$-NMR) analysis. The ratio between the integrated intensities of the $\alpha-\mathrm{CH}_{2}-\mathrm{N}$ signal of DDDMAB and the signal of the alkene protons of OA revealed that $89 \%$ of ligands are quaternary ammonium and $11 \%$ are oleate species (see also Figure S5). To achieve complete replacement of Cs-oleate with DDDMAB, we performed additional cycles of ligand exchange (see the SI for further details).,. ${ }^{1} \mathrm{H}-\mathrm{NMR}$ analysis and q-NMR analysis of the triply exchanged NCs did not reveal the presence of alkene protons resonances of $\mathrm{OA}$, thus indicating that the exchange was complete (see Figures S6-S8 of the SI). Also, repeated cycles of exchange did not compromise the colloidal stability and optical integrity of the NC dispersions (see Figure S9). The data that follow are however related to NCs exchanged only once with $\mathrm{R}_{4} \mathrm{NBr}$ species, as a single step was already sufficient to ensure the colloidal stability and near-unity QY. Compared to the Cs-oleate capped NCs, emitting at $2.41 \mathrm{eV}$ with a PLQY of $70 \pm 5 \%$, the DDDMAB capped NCs exhibited a slight blue shift (10 meV) in their PL and an increase in PLQY, which reached near-unity $100 \pm 5$ $\%$, Figure 1f). The blue shift is probably due to surface etching (see also Koscher et al.).12 Furthermore, the PL decays (Figure 1c) became faster and could be fitted to a biexponential function in which the first component had 95\% amplitude weight (very close to a single exponential), indicating that the DDDMAB capped NCs were essentially trap-free. From the fitting of the PL decays we could extract the observed lifetime and radiative $\mathrm{k}_{\mathrm{r}}$ and non-radiative $\mathrm{k}_{\mathrm{nr}}$ time constants (see Figure 1g and Table S1).

Figure 2. (a) Absorbance and PL of Cs-oleate capped and DDDMAB capped $\mathrm{CsPbBr}_{3} \mathrm{NCs}$ aged for 3 weeks under air in closed vials. (b) PLQY over time and (c) PL decays of aged samples. Thermal stability tests on (d-f) Cs-oleate capped and (g-i) DDDMAB capped 
NCs: (d-e) ${ }^{1} \mathrm{H}$ NMR spectra in toluene- $\mathrm{d}_{8}$ of Cs-oleate capped NC dispersions at various temperatures: in (d) the temperature is raised from $27^{\circ} \mathrm{C}$ to $80^{\circ} \mathrm{C}$. Then (e) the temperature is lowered to $50^{\circ} \mathrm{C}$, kept at $50^{\circ} \mathrm{C}$ for 2 hours, and lowered to $27^{\circ} \mathrm{C}$. (f) Photograph of the NMR tube after the thermal treatment. (g-i) Same analyses as for (d-f) but on DDDMAB capped NCs.

We then tested the long-term stability of the ligand exchanged samples and compared it to that of the Cs-oleate capped NCs. When stored in air for three weeks, the Csoleate capped NCs evidenced a red shift in their optical features (compare Figures 1f and 2a), their PLQY dropped from 70 to $10 \square 5 \%$ and PL lifetime increased (Figure 2b-c). Also, their morphology had changed (Figure S10). The DDDMAB capped NCs on the other hand remained unchanged in their optical features (Figure 2a-c), and their size, shape and phase were preserved (Figure S11-12). They were stable even in highly diluted dispersions (Figure S13), which is remarkable, since dilution with apolar solvents commonly promotes the detachment of ligands from the surface of halide perovskite NCs. ${ }^{22-24}$ We finally tested our ligand exchange procedure on aged Cs-oleate capped NCs, with PLQY of $30 \pm 5 \%$. Following the addition of DDDMAB to the aged NCs dispersions, the PLQY rose rapidly up to 94 $\pm 5 \%$, with no alteration in their morphology. Again, this substantial increase in the PLQY was accompanied by a significant reduction in the PL lifetimes (Figure S14).

We further compared (by means of ${ }^{1} \mathrm{H}$ NMR) the thermal stability of the Cs-oleate and DDDMAB capped NCs in toluene- $\mathrm{d}_{8}$. Cs-oleate capped NCs exhibited a significant narrowing and up-field shift of the $\alpha-\mathrm{CH}_{2}$ protons signal on increasing the temperature from 27 to $80^{\circ} \mathrm{C}$ (Figure $2 \mathrm{~d}$ ), which is indicative of the release of ligands and explains the degradation of the NCs as seen from a photograph of the NMR tube in Figure 2f. This release of ligands is not completely reversible, since the observed chemical shift remained unchanged upon reducing the temperature to $27^{\circ} \mathrm{C}$ (Figure 2e). For the DDDMAB capped NCs, upon increasing the temperature, a signal appeared at around $3.40 \mathrm{ppm}$, suggesting a partial release of ligands, however no overall chemical shift or narrowing of the peaks during the entire heat treatment cycles was observed. This means that the ligands were not desorbing and, indeed, the NCs remained stable (Figure 2 g-i). To gain further insight into the enhanced colloidal stability we investigated the DDDMAB ligand-NC surface interaction by 2D nuclear Overhauser effect spectroscopy ( ${ }^{1} \mathrm{H}$ NOESY) and DOSY (diffusion ordered spectroscopy), see Figure S15-21 for details. 2D ${ }^{1} \mathrm{H}$ NOESY, at $50{ }^{\circ} \mathrm{C}$, on solutions of free DDDMAB molecules and dispersions of DDDMAB-capped NCs exhibited positive (blue) and negative NOE cross peaks (red), respectively, which is in agreement with the characteristic of free and bound species, respectively. Heteronuclear single quantum coherence spectroscopy $\left({ }^{1} \mathrm{H}-\right.$ ${ }^{13} \mathrm{C}-\mathrm{HSQC}$ ) further confirmed that the signal of interest observed in the NOESY corresponds to the DDDMAB species. DOSY on DDDMAB-capped NCs revealed a diffusion coefficient of ca. $170 \mu \mathrm{m}^{2} / \mathrm{s}$ which is considerably smaller than that of free ligand $\left(540 \mu \mathrm{m}^{2} / \mathrm{s}\right)$, and is compatible with an estimated bound molar fraction of DDDMAB equal to $75.3 \%$. The observed diffusion coefficient in DDDMABcapped NCs is lower than what is typically observed with aliphatic ammonium ( $\left.>300 \mu \mathrm{m}^{2} / \mathrm{s}\right)$ and carboxylate $(>240$ $\left.\mu \mathrm{m}^{2} / \mathrm{s}\right)^{13}$ but is still higher than the theoretical value for tightly bound ligands for same NC size $\left(48 \mu \mathrm{m}^{2} / \mathrm{s}\right)$.

Similar results were found when the oleate capped NCs were ligand-exchanged with shorter chain quaternary ammonium ions, namely didecyl-dimethylammonium bromide (DDMAB, $\mathrm{C}_{10}$ ) and dioctyl-dimethylammonium bromide (DODMAB, $\mathrm{C}_{8}$ ). The addition of DDMAB and DODMAB to a crude solution of Cs-oleate capped $\mathrm{CsPbBr}$ NCs instantly brightened up the solution, similar to the DDDMAB $\left(\mathrm{C}_{12}\right)$ case. According to TEM (Figure S22 a, b) the ligand exchanged NCs retained their size monodispersity, and also the optical absorption profiles and XRD patterns remained basically unaffected. Again, a small blue shift (10$20 \mathrm{meV}$ ) was observed in the PL spectra of the ligand exchanged NCs (with larger shift for the shorter chain ligands). Both DDMAB and DODMAB capped NCs had near unity PLQY (Figure S22-S23 and Table S1).

To explore the origin of the enhanced PL and high stability of the NCs passivated by quaternary ammonium bromide ligands, we performed density functional theory (DFT) calculations using the cp2k package ${ }^{25}$ (see the SI for details). We built a charge-balanced nanocube of $2.4 \mathrm{~nm}$ length, terminated with $\mathrm{PbBr}_{2}$ and capped with an AX layer made of $\mathrm{CsBr}$, which in the model mimics the colloidal outer shell. At first, we focused on the ligand binding features of only one ion-pair at the surface. To build this system, we removed one $\mathrm{CsBr}$ unit from the outer $\mathrm{AX}$ shell and replaced it with a mixed organic/inorganic salt, either DDDMAB or Cs-oleate. Upon structural relaxation at the DFT/PBE level of theory, ${ }^{26}$ the quaternary ammonium bromide molecule dissociates on the surface of the NC, with the $\mathrm{Br}$ coordinating the $\mathrm{Pb}$ cation, completing the $\left[\mathrm{PbBr}_{6}\right]^{4-}$ octahedron. The anchoring of the quaternary ammonium occurs with one of the $\mathrm{N}-\mathrm{CH}_{3}$ bond sitting perpendicular to the NC surface (Figure 3a). This is in contrast with the passivation by secondary ammonium ions, for example, ${ }^{17}$ where the ligand anchors to the NC with the two methyl groups and the $\mathrm{N}$ atom lying on the same plane parallel to the NC surface (Figure S24a), orienting the long alkyl chains to increase lateral strain and disfavor a complete passivation of the surface. 

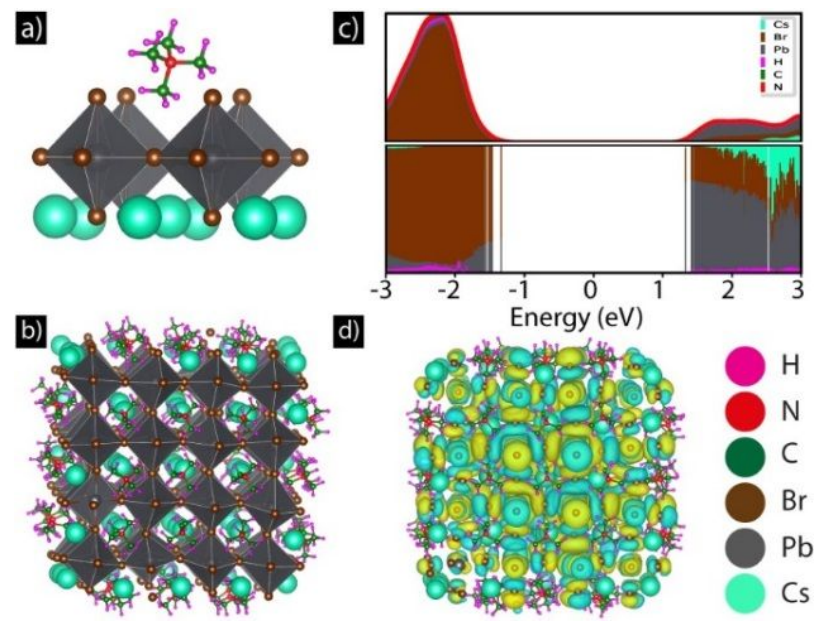

Figure 3. (a) Binding configuration of one TMAB ligand sitting in the A site. One of the $\mathrm{N}-\mathrm{CH}_{3}$ is almost perpendicular to the $\mathrm{NC}$ surface. (b) Relaxed structure of a $\mathrm{CsPbBr}_{3} \mathrm{NC}$ passivated with tetramethylammonium bromide (TMAB) with a surface concentration of 1.6 ligands $/ \mathrm{nm}^{2}$. (c) In the higher panel, a plot of the projected density of states (PDOS) of the whole NC is shown; in the lower panel each molecular orbital is depicted independently. The contribution of each atom type to molecular orbitals is shown with a different color. (d) Isosurface of the highest occupied molecular orbital (i.e., the valence band edge orbital) with a counter value of 0.02 e/Bohr ${ }^{3}$.

Additionally, we found that at the same level of theory, the calculated binding energy of DDDMAB $(48.2 \mathrm{kcal} / \mathrm{mol})$ to the NC surface is similar to that of Cs-oleate (51.3 $\mathrm{kcal} / \mathrm{mol}$ ). These values are in line with the binding energies computed for primary ammonium bromides (45.3 $\mathrm{kcal} / \mathrm{mol})^{13}$ or zwitterionic ligands such as sulfobetaine $(41.0 \mathrm{kcal} / \mathrm{mol})^{16}$, indicating that the interaction between the NC and the ligand is dominated by electrostatic interactions, irrespectively of the type of anchoring group employed. Positioning one ligand, however, takes into account only the local interaction of the binding group to the surface, neglecting ligand-to-ligand interactions. To include this effect, we decided to fully passivate one of the facets of our NC model with either DDDMAB or Cs-oleate, attaining a ligand surface concentration of 2.8 ligands $/ \mathrm{nm}^{2}$ (Figure S24-c), chosen to be higher than the 1.6 attributed in the experiment. This choice, however, provides an upper limit to the steric interaction between the ligands. In this configuration, we find that the steric repulsion between the ligands decreases the binding energy to $39.1 \mathrm{kcal} / \mathrm{mol}$ per ligand in the case of Cs-oleate and to $36.6 \mathrm{kcal} / \mathrm{mol}$ for DDDMAB. These values again indicate that these types of ligands still remain tightly bound to the surface with no particular difference between DDDMAB and Cs-oleate, with the latter being even slightly stronger. This result implicitly suggests that the main origin of the higher binding affinity of DDDMAB to the NC surface found in the experiments, compared to Cs-oleate, might be ascribed to solvent-ligand interactions, which are not captured by the calculations. A full exploration of this effect from the computational standpoint is quite involved and will be assessed in future theoretical works.

Finally, to assess the structural tolerance to form defects for ammonium passivated systems we replaced $\mathrm{CsBr}$ units from all six facets with tetramethyl ammonium bromide (TMAB) to attain an overall surface concentration of 1.6 ligands $/ \mathrm{nm}^{2}$ in order to look at the effect on the computed electronic structure. We chose TMAB to facilitate calculations as the system size grows significantly with the number of ligands. Despite the moderate passivation, after structural relaxation (Figure $3 \mathrm{~b}$ ), the electronic structure of this system is free of midgap states (Figure 3c) as also highlighted by the complete wavefunction delocalization of the highest occupied orbital, which is usually prone to the formation of trap states (Figure $3 \mathrm{~d}$ ). ${ }^{27}$

We also ran a series of ligand exchange trials using various toluene-soluble secondary, tertiary and quaternary ammonium bromides: didodecyl ammonium bromide (DDAB), dimethyldodecyl ammonium bromide (DMDDAB), and tetraoctyl ammonium bromide (TOAB) (see Figure S25). A significant increase in PL was observed after treatment with aforementioned ligands, however all the samples degraded after two weeks of storage under ambient conditions. We ascribe the degradation of samples treated with secondary and tertiary ammonium bromides to the dissociation of these ligands, while the degradation of sample treated with $\mathrm{TOAB}$ is most likely due to the fact that the positive charge in TOAB is too far from the NC surface to provide adequate stability.

In summary, we have reported a cation and anion simultaneous exchange strategy involving the displacement of both ionic ligands on Cs-oleate capped NCs. Cesium oleate ligands coating the surface of as-synthesized $\mathrm{CsPbBr}_{3} \mathrm{NCs}$ were exchanged with various quaternary ammonium bromide $\left(\mathrm{R}_{4} \mathrm{NBr}\right)$ species, and ligand exchange was confirmed by extensive NMR characterization. The successful exchange ensured colloidal, structural and morphological integrity of the NCs and additionally pushed their PLQY to near unity, which was preserved after washing with polar solvents, over three weeks of storage in air, and even after heating. We attribute this remarkable set of properties to the stronger binding affinity of $\mathrm{R}_{4} \mathrm{NBr}$ to the surface of the NCs. Ligand exchange driven exclusive passivation with short chain quaternary ammonium ions (for example $\mathrm{C}_{8}$ ) will be particularly appealing for using these NCs in light emission related technologies.

\section{ASSOCIATED CONTENT}

Supporting Information. Details on synthesis, on characterization of the materials and on computations.

\section{AUTHOR INFORMATION}

\section{Corresponding Author}

*Ivan Infante ivan.infante@iit.it

*Liberato Manna liberato.manna@iit.it

ACKNOWLEDGMENT 
We acknowledge funding from the European Union under grant agreement n. 614897 (ERC Grant TRANS-NANO). I.I. acknowledges Dutch NWO for financial support under the Vidi scheme (Grant n. 723.013.002).

\section{REFERENCES}

(1) Zhang, W.; Eperon, G. E.; Snaith, H. J. Metal Halide Perovskites for Energy Applications. Nat. Energy 2016, 1, 16048.

(2) Sutherland, B. R.; Sargent, E. H. Perovskite Photonic Sources. Nat. Phot. 2016, 10, 295-302.

(3) Yang, D.; Li, X.; Zeng, H. Surface Chemistry of All Inorganic Halide Perovskite Nanocrystals: Passivation Mechanism and Stability. Adv. Mater. Int. 2018, 5, 1701662.

(4) Huang, J.; Lai, M.; Lin, J.; Yang, P. Rich Chemistry in Inorganic Halide Perovskite Nanostructures. Adv. Mater. 2018, 1802856.

(5) Imran, M.; Caligiuri, V.; Wang, M.; Goldoni, L.; Prato, M.; Krahne, R.; De Trizio, L.; Manna, L. Benzoyl Halides as Alternative Precursors for the Colloidal Synthesis of Lead-Based Halide Perovskite Nanocrystals. J. Am. Chem. Soc. 2018, 140, 2656-2664.

(6) Protesescu, L.; Yakunin, S.; Bodnarchuk, M. I.; Krieg, F.; Caputo, R.; Hendon, C. H.; Yang, R. X.; Walsh, A.; Kovalenko, M. V. Nanocrystals of Cesium Lead Halide Perovskites $\left(\mathrm{CsPbX}_{3}, \mathrm{X}=\mathrm{Cl}, \mathrm{Br}\right.$, and I): Novel Optoelectronic Materials Showing Bright Emission with Wide Color Gamut. Nano Lett. 2015, 15, 3692-3696.

(7) Bodnarchuk, M. I.; Boehme, S. C.; ten Brinck, S.; Bernasconi, C.; Shynkarenko, Y.; Krieg, F.; Widmer, R.; Aeschlimann, B.; Günther, D.; Kovalenko, M. V., et al. Rationalizing and Controlling the Surface Structure and Electronic Passivation of Cesium Lead Halide Nanocrystals. ACS Energy Lett. 2018, 4, 63-74.

(8) De Roo, J.; Ibanez, M.; Geiregat, P.; Nedelcu, G.; Walravens, W.; Maes, J.; Martins, J. C.; Van Driessche, I.; Kovalenko, M. V.; Hens, Z. Highly Dynamic Ligand Binding and Light Absorption Coefficient of Cesium Lead Bromide Perovskite Nanocrystals. ACS Nano 2016, 10, 2071-2081.

(9) Smock, S. R.; Williams, T. J.; Brutchey, R. L. Quantifying the Thermodynamics of Ligand Binding to $\mathrm{CsPbBr}_{3}$ Quantum Dots. Angew. Chem. Int. Ed. Engl 2018, 57, 11711-11715.

(10) Wu, Y.; Wei, C.; Li, X.; Li, Y.; Qiu, S.; Shen, W.; Cai, B.; Sun, Z.; Yang, D.; Deng, Z. In Situ Passivation of $\mathrm{PbBr}_{6}{ }^{4-}$ Octahedra toward Blue Luminescent $\mathrm{CsPbBr}_{3}$ Nanoplatelets with Near 100\% Absolute Quantum Yield. ACS Energy Lett. 2018, 3, 2030-2037.

(11) Ahmed, T.; Seth, S.; Samanta, A. Boosting the Photoluminescence of $\mathrm{CsPbX}_{3}(\mathrm{X}=\mathrm{Cl}, \mathrm{Br}, \mathrm{I})$ Perovskite Nanocrystals Covering a Wide Wavelength Range by Post-Synthetic Treatment with Tetrafluoroborate Salts. Chem. Mater. 2018, 30, 3633-3637.

(12) Koscher, B. A.; Swabeck, J. K.; Bronstein, N. D.; Alivisatos, A. P. Essentially Trap-Free $\mathrm{CsPbBr}_{3}$ Colloidal
Nanocrystals by Postsynthetic Thiocyanate Surface Treatment. J. Am. Chem. Soc. 2017, 139, 6566-6569.

(13) Almeida, G.; Ashton, O. J.; Goldoni, L.; Maggioni, D.; Petralanda, U.; Mishra, N.; Akkerman, Q. A.; Infante, I.; Snaith, H. J.; Manna, L. The Phosphine Oxide Route toward Lead Halide Perovskite Nanocrystals. J. Am. Chem. Soc. 2018, 140, 14878-14886.

(14) Li, X.; Dar, M. I.; Yi, C.; Luo, J.; Tschumi, M.; Zakeeruddin, S. M.; Nazeeruddin, M. K.; Han, H.; Gratzel, M. Improved Performance and Stability of Perovskite Solar Cells by Crystal Crosslinking with Alkylphosphonic Acid $\omega$-ammonium Chlorides. Nat. Chem. 2015, 7, 703711.

(15) Koh, W.-k.; Park, S.; Ham, Y. Phosphonic Acid Stabilized Colloidal $\mathrm{CsPbX}_{3}(\mathrm{X}=\mathrm{Br}, \quad$ I) Perovskite Nanocrystals and Their Surface Chemistry. Chem. Sel. 2016, 1, 3479-3482.

(16) Krieg, F.; Ochsenbein, S. T.; Yakunin, S.; Ten Brinck, S.; Aellen, P.; Suess, A.; Clerc, B.; Guggisberg, D.; Nazarenko, O.; Shynkarenko, Y., et al. Colloidal CsPbX ${ }_{3}$ (X $=\mathrm{Cl}, \mathrm{Br}, \mathrm{I})$ Nanocrystals 2.0: Zwitterionic Capping Ligands for Improved Durability and Stability. ACS Energy Lett. 2018, 3, 641-646.

(17) Imran, M.; Ijaz, P.; Baranov, D.; Goldoni, L.; Petralanda, U.; Akkerman, Q.; Abdelhady, A. L.; Prato, M.; Bianchini, P.; Infante, I., et al. Shape-Pure, Nearly Monodispersed $\mathrm{CsPbBr}_{3}$ Nanocubes Prepared Using Secondary Aliphatic Amines. Nano Lett. 2018, 18, 78227831.

(18) Zheng, X.; Chen, B.; Dai, J.; Fang, Y.; Bai, Y.; Lin, Y.; Wei, H.; Zeng, Xiao C.; Huang, J. Defect Passivation in Hybrid Perovskite Solar Cells using Quaternary Ammonium Halide Anions and Cations. Nat. Energy 2017, 2, 17102.

(19) Pan, J.; Quan, L. N.; Zhao, Y.; Peng, W.; Murali, B.; Sarmah, S. P.; Yuan, M.; Sinatra, L.; Alyami, N. M.; Liu, J., et al. Highly Efficient Perovskite-Quantum-Dot LightEmitting Diodes by Surface Engineering. Adv. Mater. 2016, 28, 8718-8725.

(20) Song, J.; Fang, T.; Li, J.; Xu, L.; Zhang, F.; Han, B.; Shan, Q.; Zeng, H. Organic-Inorganic Hybrid Passivation Enables Perovskite QLEDs with an EQE of $16.48 \%$. Adv. Mater. 2018, 1805409.

(21) Dang, Z.; Shamsi, J.; Palazon, F.; Imran, M.; Akkerman, Q. A.; Park, S.; Bertoni, G.; Prato, M.; Brescia, R.; Manna, L. In Situ Transmission Electron Microscopy Study of Electron Beam-Induced Transformations in Colloidal Cesium Lead Halide Perovskite Nanocrystals. ACS Nano 2017, 11, 2124-2132.

(22) Udayabhaskararao, T.; Houben, L.; Cohen, H.; Menahem, M.; Pinkas, I.; Avram, L.; Wolf, T.; Teitelboim, A.; Leskes, M.; Yaffe, O. A Mechanistic Study of Phase Transformation in Perovskite Nanocrystals Driven by Ligand Passivation. Chem. Mater. 2017, 30, 84-93.

(23) Di Stasio, F.; Imran, M.; Akkerman, Q. A.; Prato, M.; Manna, L.; Krahne, R. Reversible ConcentrationDependent Photoluminescence Quenching and Change 
of Emission Color in $\mathrm{CsPbBr}_{3}$ Nanowires and Nanoplatelets. J. Phys. Chem. Lett. 2017, 8, 2725-2729. (24) Grabolle, M.; Spieles, M.; Lesnyak, V.; Gaponik, N.; Eychmüller, A.; Resch-Genger, U. Determination of the Fluorescence Quantum Yield of Quantum Dots: Suitable Procedures and Achievable Uncertainties. Anal. Chem. 2009, 81, 6285-6294.

(25) Hutter, J.; Iannuzzi, M.; Schiffmann, F.; VandeVondele, J. CP2K: Atomistic Simulations of
Condensed Matter Systems. Wiley Interdiscip. Rev. Comput. Mol. Sci. 2014, 4, 15-25.

(26) Perdew, J. P.; Burke, K.; Ernzerhof, M. Generalized Gradient Approximation Made Simple. Phys. Rev. lett. 1996, 77, 3865.

(27) ten Brinck, S.; Infante, I. Surface Termination, Morphology, and Bright Photoluminescence of Cesium Lead Halide Perovskite Nanocrystals. ACS Energy Lett. 2016, 1, 1266-1272. 
2

3

4

5 